\title{
Enzymatic Synthesis of Multiple Spin-Labeled DNA**
}

\section{Samra Obeid, Maxim Yulikov, Gunnar Jeschke, and Andreas Marx*}

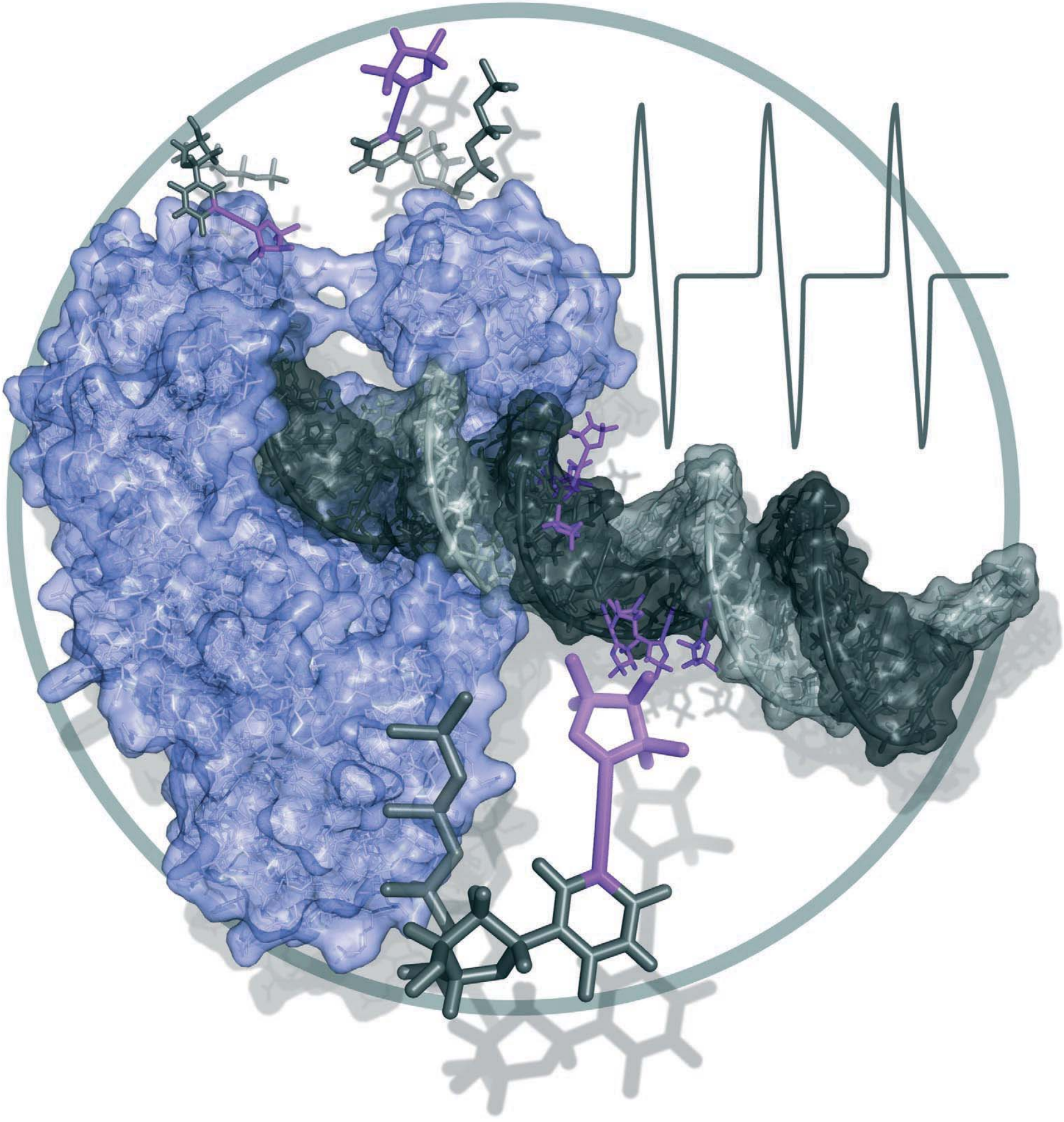


Electron paramagnetic resonance (EPR) spectroscopy is a widespread technique for the study of the organizational and dynamic properties of biological macromolecules. Many of these applications depend on the sensitivity of nitroxide labels ${ }^{[1]}$ to dynamics on the picosecond to microsecond time scales and on the ability to measure distances between such labels on the nanometer length scale. ${ }^{[2]}$ These techniques are applicable in disordered systems, more sensitive than NMR measurements, and provide more detailed information than techniques based on optical excitation. Most biomacromole cules are diamagnetic in their native states and thus do not have background EPR signals. Thus, spin labeling techniques can selectively address sites of interest in large molecules and complex assemblies. ${ }^{[3]}$ Attachment through a rigid linker is necessary to minimize the effect of the motion of the spin label on the spectrum and at the same time to maximize the backbone dynamics of the biomacromolecule. ${ }^{[4]}$ Such rigid linkers are also favorable for distance measurements as they lead to narrower distance distributions and thus to smaller uncertainties in translating distances between labels to structural models. However, the use of rigid linkers generates the risk of perturbating the native structure because the label cannot adapt to the steric requirements of its environment. For this reason, labeling strategies have to be designed and tested with great care. ${ }^{[5]}$

Recently, EPR spectroscopy was applied extensively in studies on the structures and dynamics of nucleic acids. ${ }^{[1,6]}$ Since nucleic acids do not contain any natural paramagnetic centers, spin labels have to be introduced prior to EPR investigations. Several methods have previously been estab lished for the introduction of a paramagnetic center, for example, a stable nitroxide, at a specific site in DNA. Such spin labels were introduced either by employment of a spin labeled building block during automatic DNA synthesis, ${ }^{[7]}$ or functionalized building blocks were introduced into the growing DNA first and subsequently coupled to a spin label on a solid support, for example, by employment of palladium catalyzed coupling reactions. ${ }^{[8]}$ Single labeled oligonucleoti des with relatively short lengths have been synthesized by these techniques. However, the length of the oligonucleotides and their degree of modification is restricted by the inherent limitations of automatic DNA synthesis. To the best of our knowledge, multiple site specific incorporation of spin labels into DNA by using these methods has not as yet been demonstrated. Other approaches are based on the incorpo ration of additional functionalities in nucleic acids that are

[*] S. Obeid, Dr. M. Yulikov, ${ }^{[+]}$Prof. Dr. G. Jeschke, ${ }^{[+]}$Prof. Dr. A. Marx Department of Chemistry and

Konstanz Research School Chemical Biology University of Konstanz

Universitätsstrasse 10, 78457 Konstanz (Germany)

Fax: $(+49) 7531885140$

Email: andreas.marx@uni konstanz.de

$\left.{ }^{+}\right]$Present address:

Department of Chemistry, ETH Zurich

8093 Zurich (Switzerland).

[**] We gratefully acknowledge funding by the DFG. conjugated with suitably activated spin probes after their chemical or enzymatic synthesis. ${ }^{[9]}$ These methods allow the incorporation of multiple probes into one molecule. However, short linkers between the nucleic acid and the paramagnetic center are still rather flexible, which have disadvantages for the interpretation of the subsequent EPR spectra.

Here we present the site specific introduction of spin labels into DNA by using suitably modified nucleoside triphosphates as building blocks in template directed reac tions catalyzed by DNA polymerases. The synthesis of multiple spin labeled, long DNA oligonucleotides is feasible by using this approach. We found that DNA polymerases of eukaryotic, prokaryotic, and archaic origin can accommodate the employment of spin labeled nucleotides as building blocks for DNA synthesis.

We first carried out the synthesis of the spin labeled nucleoside triphosphates $\mathbf{1}$ that contain nitroxide based para magnetic centers connected to the nucleobase (Scheme 1).
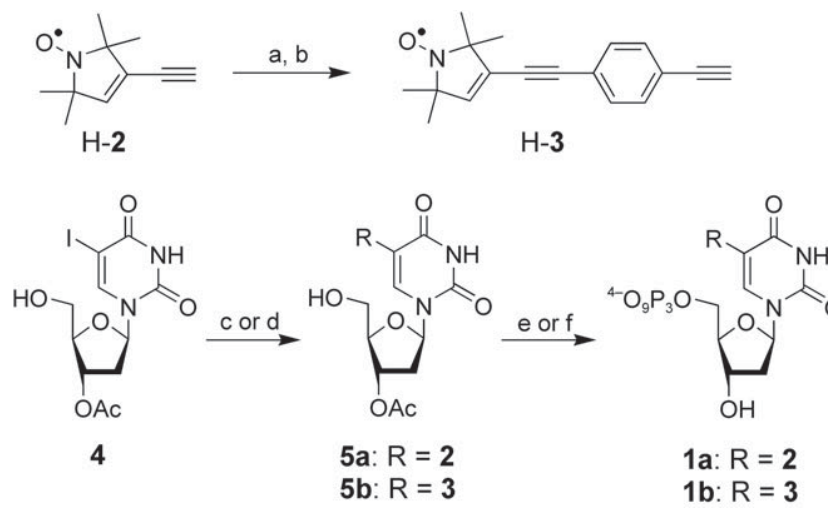

Scheme 1. Synthesis of spin labeled TTP analogues 1. Conditions and reagents: a) (2 (4 iodophenyl)ethynyl)trimethylsilane, $\left[\mathrm{Pd}\left(\mathrm{PPh}_{3}\right)_{4}\right], \mathrm{Cul}$, DMF, microwave, $15 \mathrm{~min}, 100^{\circ} \mathrm{C}, 64 \%$; b) tetrabutylammonium fluo ride, $\mathrm{CH}_{2} \mathrm{Cl}_{2}, 5 \mathrm{~min}, 0^{\circ} \mathrm{C}, 83 \%$; c) $\mathrm{H} 2$, [Pd $\left.\left(\mathrm{PPh}_{3}\right)_{4}\right]$, Cul, DMF, $43 \%$ (5a); d) H 3, [Pd(PPh $\left.)_{4}\right], \mathrm{Cul}, \mathrm{DMF}, 67 \%$ (5 b); e) 2 chloro 4H 1,3,2 benzodioxaphosphorin 4 one, $\left(n \mathrm{Bu}_{3} \mathrm{NH}\right)_{2} \mathrm{H}_{2} \mathrm{P}_{2} \mathrm{O}_{7}, \mathrm{I}_{2}$, pyridine, $\mathrm{H}_{2} \mathrm{O}$, then $\mathrm{NH}_{3}, 31 \%$ (1 a); f) 2 chloro $4 \mathrm{H} \mathrm{1,3,2} \mathrm{benzodioxaphosphorin} 4$ one, $\left(n \mathrm{Bu}_{3} \mathrm{NH}\right)_{2} \mathrm{H}_{2} \mathrm{P}_{2} \mathrm{O}_{7}$, then $\mathrm{I}_{2}$, pyridine, $\mathrm{H}_{2} \mathrm{O}$, then $\mathrm{NH}_{3}, 31 \%(1 \mathrm{~b})$.

We chose modification at the $\mathrm{C} 5$ position in $2^{\prime}$ deoxyuridine since modifications at this position do not significantly interfere with Watson Crick base pairing. Furthermore, it has been shown that DNA polymerases are able to tolerate modifications at this position at least to a certain extent, as seen in the substitution of thymidine with other base analogues. ${ }^{[10,11]}$ In addition, the palladium catalyzed cross cou pling of alkynes to the respective 5 iodo $2^{\prime}$ deoxyuridine analogues has been demonstrated. ${ }^{[12,13]}$ To assess the effect of the length of the linker that connects the spin label with the nucleotide on the ability of DNA polymerases to accept $\mathbf{1}$ we synthesized alkyne modified nitroxide $\mathrm{H} \mathbf{3}$ starting from known $\mathrm{H} 2^{[7 b, 14]}$ by a Sonogashira cross coupling reaction. Nitroxides $\mathrm{H}$ $\mathbf{2}$ and $\mathrm{H} 3$ were coupled with the protected 5 iodo $2^{\prime}$ deoxyur idine derivative $\mathbf{4}$ to yield $\mathbf{5}$, which was subsequently trans formed into the nucleoside triphosphates $\mathbf{1}^{[15]}$

We next studied the action of nucleoside triphosphates $\mathbf{1}$ on DNA polymerases by investigating the propensity of the 
Klenow fragment of E. coli DNA polymerase I $\left(3^{\prime} \rightarrow 5^{\prime}\right.$ exonuclease deficient variant, $\mathrm{KF}($ exo $))$ and human DNA polymerase $\beta(\operatorname{Pol} \beta)$ to accept $\mathbf{1}$ in primer extension experi ments. First we used a $5^{\prime}$ end ${ }^{32} \mathrm{P}$ labeled 23 nucleotide primer and 35 nucleotide template complex that leads to incorpo ration of a thymidine analogue at position 27 after incorpo ration of three nucleotides (Figure $1 \mathrm{a}$ ). We found for both

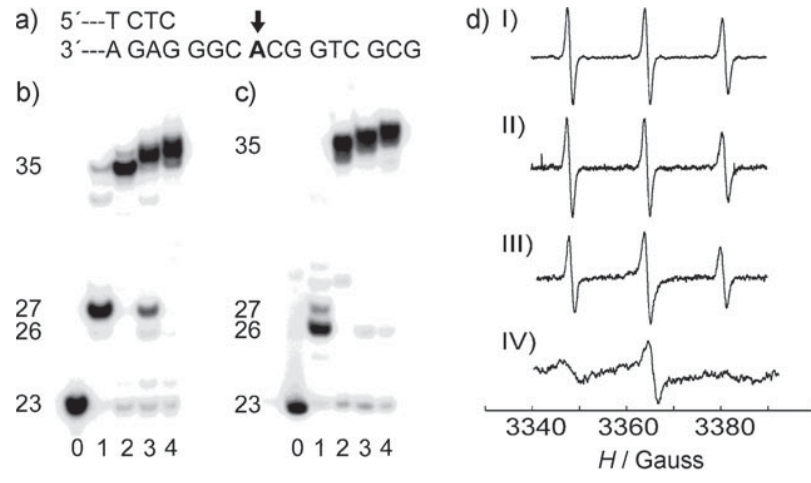

Figure 1. Incorporation of $\mathbf{1} \mathbf{a}, \mathbf{b}$ and EPR investigations. a) Partial sequences of the primer and template. b) Primer extension studies employing $K F($ exo ): lane 0: primer only, lane 1: primer extension performed in the presence of dATP, dGTP, and dCTP, but without any TTP analogue; lane 2: as lane 1, but in the presence of TTP; lane 3 : as lane 1, but in the presence of $1 \mathrm{a}$; lane 4 : as lane 1, but in the presence of 1 b. c) As in (b), but with the use of Pol $\beta$ instead of KF(exo ). d) EPR spectra of $1 \mathbf{a}(\mathrm{I}), \mathbf{1} \mathbf{b}$ (II), the product derived from the reaction in (b), lane 3 , by using 1 a (III), and the product derived from the reaction in (b), lane 4, by using $\mathbf{1}$ b (IV). Further experimental details are provided in the Supporting Information.

enzymes that primer elongation is paused at positions 26 or 27 when the reactions were performed in the presence of only dATP, dGTP, and dCTP (Figure 1 b, c; lanes 1). When all four natural dNTPs were added, both enzymes resulted in the formation of the full length product (Figure 1b, c; lanes 2). When the natural thymidine triphosphate (TTP) was sub stituted by $\mathbf{1 a}$ or $\mathbf{1 b}$, respectively, reaction products were formed that migrated slower in denaturing polyacrylamide gel electrophoresis (PAGE) than the original band corresponding to the full length products derived from experiments with TTP (Figure 1b, c; lanes 3 and 4). The retardation can be explained by the additional bulk of the modifications. Similar effects have been reported before. ${ }^{[10]}$ Interestingly, significant pausing bands were observed at position 27 when 1 a was employed in the $\mathrm{KF}$ (exo ) catalyzed reaction (Figure $1 \mathrm{~b}$, lane 3 ). This finding indicates that further extension is some how hampered after incorporation of $\mathbf{1 a}$. No such bands were observed when nucleotide $\mathbf{1 b}$ was employed, which indicates that the bulky nitroxide group is better tolerated by this enzyme when placed on an extended linker. After purification of the enzymatically synthesized DNA by HPLC, the CD spectra indicates that the modified oligonucleotides adopt a similar overall B form conformation as the unmodified strands (see the Supporting Information).

The successful incorporation of the nucleotides derived from $\mathbf{1} \mathbf{a}$ and $\mathbf{1 b}$ was further verified by ESI mass spectrom etry and EPR spectroscopy. The EPR spectra of $\mathbf{1} \mathbf{a}$ and $\mathbf{1 b}$
(Figure $1 \mathrm{~d}$, traces I and II) can be fitted well by assuming an isotropic rotational diffusion in the fast regime, ${ }^{[16]}$ with rotational correlation times $\tau_{\mathrm{c}}$ of 57 and $95 \mathrm{ps}$, respectively (see the Supporting Information). The increase in the value of $\tau_{\mathrm{c}}$ with increasing linker length is in line with expectations. After the incorporation (traces III and IV), the model of isotropic rotational diffusion no longer fits the spectra. The relative line intensities are consistent with a preferred motion about the linker backbone axis. Molecular rotation perpen dicular to this axis is slower, but is still well within the fast regime for the shorter linker (trace III). This result indicates rotation about the DNA helix axis on a sub nanosecond time scale. This rotation is slowed down for the longer linker (trace IV) and approaches the nanosecond time scale. As the modeling of the spectral line shapes are ambiguous for anisotropic rotational diffusion in the fast regime, we refrain from deriving rotational diffusion tensors.

Encouraged by these initial findings, we next investigated whether multiple spin labels could be incorporated within one helix by DNA polymerases. For this we designed two templates in a way that they code for the incorporation of a spin labeled nucleotide at every second or every fourth nucleotide. A third template was designed so that 11 adjacent 2 ' deoxyadenosine residues code for the incorporation of 11 spin labeled nucleotides in a row and form one entire DNA helix turn of spin labels. Preliminary studies of several DNA polymerases indicated that commercially available Thermi nator DNA polymerase (A485L mutant of Thermococcus species $9^{\circ} \mathrm{N}$ DNA polymerase) is most proficient in processing multiple spin labeled nucleotides $\mathbf{1 a}$ and $\mathbf{1 b}$. Furthermore, it turned out that this enzyme accepted $\mathbf{1} \mathbf{a}$ better than $\mathbf{1 b}$. Thus, for further studies we used 1 a exclusively. Figure 2 shows the results obtained in multiple incorporation experiments by using Therminator DNA polymerase. Full length products were obtained in all cases when natural TTP was substituted by 1a. Again, slower migrating products were observed by PAGE analysis when the bulkier 1a was used instead of natural TTP.

Interestingly, Therminator DNA polymerase is proficient in incorporating 11 adjacent modified nucleotides of $\mathbf{1 a}$, thereby resulting in one entire DNA helical turn decorated with multiple spin systems (Figure $2 \mathrm{c}$ ). The reaction product was purified by HPLC and further characterized by thermal denaturation $\left(T_{\mathrm{m}}\right)$ measurements as well as by CD and EPR spectroscopy. The CD spectrum indicates that the intensively modified DNA bearing 11 adjacent spin probes indeed adopts an overall B form conformation similar to the unmodified DNA (see the Supporting Information). In addition, the $T_{\mathrm{m}}$ measurements indicate only a small effect of the modifi cations on the stability of the duplex (see the Supporting Information).

Surprisingly, the EPR spectrum (Figure 3) indicates a reduced anisotropy of rotational diffusion compared to the singly labeled DNA. However, unlike in the spectrum of $\mathbf{1 a}$, the low field line still has a significantly smaller amplitude than the center line, which demonstrates that the motion is not completely isotropic. The absence of line shape effects arising from through space exchange coupling, except for the additional homogeneous line broadening compared to the 


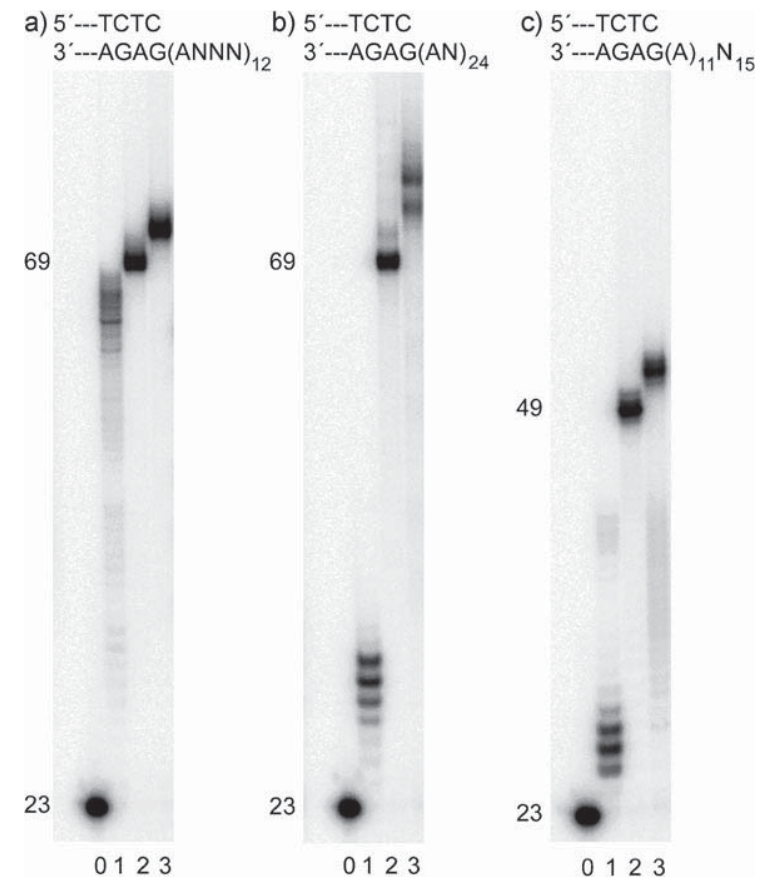

Figure 2. Multiple spin labeling under Therminator DNA polymerase catalysis and incorporation of $1 \mathbf{a}$. The primer template sequences employed are indicated above each panel. Lanes 0: primer only; lanes 1: primer extension performed in the presence of dATP, dGTP, and dCTP, but without any TTP analogue; lanes 2: as in lanes 1, but in the presence of TTP; lanes 3 : as in lanes 1, but in the presence of $1 \mathrm{a}$.

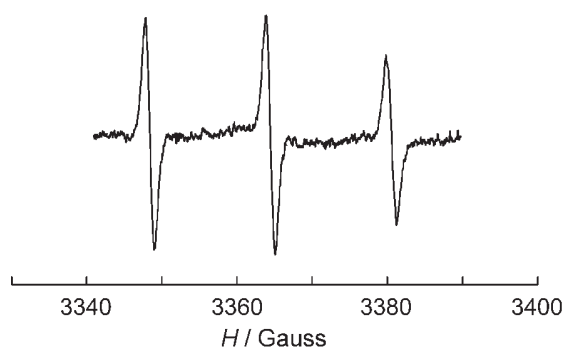

Figure 3. EPR spectra of a 49nt DNA duplex containing 11 adjacent spin labeled nucleotides derived from $1 \mathrm{a}$.

spectra of $\mathbf{1 a}$, is feasible for a rigid structure. Solid state spectra that would have been conclusive about the presence or absence of dipole dipole coupling between the spins could not be obtained with the currently available sample concen tration. We do find, however, that the intensity of the spectrum is consistent with the presence of 11 spin labels per DNA molecule at the given DNA concentration. The EPR spectra are thus also in agreement with the proposed structure, although it is unclear at present why the motion about the helix axis is faster for the multiply labeled $49 \mathrm{mer}$ than for the singly labeled $35 \mathrm{mer}$.

In summary, we have demonstrated for the first time the synthesis of spin labeled nucleoside triphosphates $\mathbf{1}$ and their proficiency to be accepted as substrates by DNA polymerases. DNA polymerases of eukaryotic, prokaryotic, and archaic origin are competent in the employment of spin labeled nucleotides as surrogates of natural building blocks in enzy matic DNA synthesis. This finding opens up new opportunities for further applications, for example, for in vivo spin labeling or the generation of complex multiple spin systems, which might be useful for DNA based nanobiotechnology. Further inves tigations along these lines are in progress.

Received: May 17, 2008

Published online: July 31, 2008

Keywords: DNA polymerase - DNA - EPR spectroscopy · nucleotides $\cdot$ spin labels

[1] Spin Labeling: The Next Millennium (Biol. Magn. Reson. 1998, 14).

[2] a) G. Jeschke, Y. Polyhach, Phys. Chem. Chem. Phys. 2007, 9, 1895; b) O. Schiemann, T. F. Prisner, Q. Rev. Biophys. 2007 40, 1.

[3] W. L. Hubbell, D. S. Cafiso, C. Altenbach, Nat. Struct. Biol. 2000, 7,735 .

[4] A. Spaltenstein, B. H. Robinson, P. B. Hopkins, J. Am. Chem. Soc. 1988, 110, 1299.

[5] O. Schiemann, N. Piton, J. Plackmeyer, B. E. Bode, T. F. Prisner, J. W. Engels, Nat. Protocols 2007, 2, 904.

[6] a) P. Z. Qin, I. S. Haworth, Q. Cai, A. K. Kusnetzow, G. P. G. Grant, E. A. Price, G. Z. Sowa, A. Popova, B. Herreros, H. He, Nat. Protocols 2007, 2, 2354; b) R. Ward, D. J. Keeble, H. El Mkami, D. G. Norman, ChemBioChem 2007, 8, 1957; c) N. Barhate, P. Cekan, A. P. Massey, S. T. Sigurdsson, Angew. Chem. 2007, 119, 2709; Angew. Chem. Int. Ed. 2007, 46, 2655.

[7] a) A. Spaltenstein, B. H. Robinson, P. B. Hopkins, J. Am. Chem. Soc. 1988, 110, 1299; b) A. Spaltenstein, B. H. Robinson, P. B. Hopkins, Biochemistry 1989, 28, 9484.

[8] a) O. Schiemann, N. Piton, Y. Mu, G. Stock, J. W. Engels, T. F. Prisner, J. Am. Chem. Soc. 2004, 126, 5722; b) O. Schiemann, N. Piton, J. Plackmeyer, B. E. Bode, T. F. Prisner, J. W. Engels, Nucleosides Nucleotides Nucleic Acids 2005, 24,771; c) N. Piton, Y. Mu, G. Stock, T. F. Prisner, O. Schiemann, J. W. Engels, Nucleic Acids Res. 2007, 35, 3128.

[9] a) P. W. Langenmeier, A. M. Bobst, Arch. Biochem. Biophys. 1981, 208, 205; b) A. M. Bobst, S. C. Kao, R. C. Toppin, J. C. Ireland, I. E. Thomas, J. Mol. Biol. 1984, 173, 63.

[10] a) O. Thum, S. Jäger, M. Famulok, Angew. Chem. 2001, 113, 4112; Angew. Chem. Int. Ed. 2001, 40, 3990; b) S. Jäger, M. Famulok, Angew. Chem. 2004, 116, 3399; Angew. Chem. Int. Ed. 2004, 43, 3337; c) S. Jäger, G. Rasched, H. Kornreich Leshem, M. Engeser, O. Thum, M. Famulok, J. Am. Chem. Soc. 2005, 127, 15071; d) P. Čapek, H. Cahová, R. Pohl, M. Hocek, C. Gloeckner, A. Marx, Chem. Eur. J. 2007, 13, 6115.

[11] H. Sawai, A. N. Ozaki, F. Satoh, T. Ohbayashi, M. M. Masud, H. Osaki, Chem. Commun. 2001, 2604.

[12] a) M. J. Robbins, P. Barr, Tetrahedron Lett. 1981, 22, 421; b) F. W. J. Hobbs, J. Org. Chem. 1989, 54, 3420; c) J. D. Kahl, M. M. Greenberg, J. Am. Chem. Soc. 1999, 121, 597.

[13] a) L. A. Agrofoglio, I. Gillaizeau, Y. Saito, Chem. Rev. 2003, 103, 1875; b) R. Chinhilla, C. Najera, Chem. Rev. 2007, 107, 874.

[14] S. W. Stork, M. W. Makinen, Synthesis 1999, 1309.

[15] J. Ludwig, F. Eckstein, J. Org. Chem. 1989, 54, 631.

[16] J. H. Freed, G. K. Fraenkel, J. Chem. Phys. 1963, 39, 326. 